\title{
Genomic evidence of multiple SARS-CoV-2 introductions into
}

\section{Morocco [version 1; peer review: 1 approved with}

\section{reservations]}

\author{
Bouchra Chaouni ${ }^{1}$, Imane Allali ${ }^{1}$, Sofia Sehli2,3, Wajih Rhalem ${ }^{4}$, \\ Abdellah Idrissi Azami (D)2,3, Nihal Habib2,3, Salsabil Hamdi'5, Lahcen Wakrim5, \\ Abderrahmane Bakkali (iD6, Mustapha Mahmoud7, Laila Bouguenouch7, \\ Najib Al Idrissi ${ }^{8}$, Youssef Bakri ${ }^{1}$ Saaïd Amzazi ${ }^{1}$, Said Barrijal ${ }^{9}$, Hassan Ghazal (iD2
}

\footnotetext{
${ }^{1}$ Faculty of Sciences, University Mohammed V, Rabat, Morocco

${ }^{2}$ National Center for Scientific and Technical Research (CNRST), Rabat, Morocco

${ }^{3}$ Faculty of Medicine \& Pharmacy, University Mohammed V, Rabat, Morocco

${ }^{4}$ National High School for Arts and Professions, University Mohammed V, Rabat, Morocco

Institut Pasteur Morocco, Casablanca, Morocco

${ }^{6}$ Faculty of Medicine \& Pharmacy, University Ibn Zohr, Agadir, Morocco

${ }^{7}$ Faculty of Medicine \& Pharmacy, University Sidi Mohammed Ben Abdellah, Fes, Morocco

${ }^{8}$ Faculty of Medicine, University Mohammed VI for Health Sciences, Casablanca, Morocco

${ }^{9}$ Faculty of Sciences and Technology, University Abdelmalek Essaâdi, Tangier, Morocco
}

\section{V1 First published: $06 \mathrm{Jul} 2020,9: 679$ \\ https://doi.org/10.12688/f1000research.24810.1}

Latest published: 24 Aug 2020, 9:679

https://doi.org/10.12688/f1000research.24810.2

\section{Abstract}

Background: The recent emergence of a novel coronavirus (SARSCoV-2) has caused serious public health concerns due to its rapid dissemination worldwide. A total of 8,931 positive cases had been reported in Morocco by the $16^{\text {th }}$ of June 2020.

Methods: To better understand the SARS-CoV-2 pandemic in this North African country, we analyzed the complete genome sequences of the virus related to Morocco by constructing a phylogenetic tree and creating a variant network using the available Moroccan and other sequences in dedicated databases.

Results: Phylogenetic and variant network analyses of SARS-CoV-2 strains from early patients with COVID-19 in Morocco showed multiple spatiotemporal introductions from Italy (ten), France (seven), Spain (one) and Portugal (one). This is consistent with the assumption that the early infections in Morocco were imported, mainly from Europe. The 17 virus strains form two independent phylogenetic clusters and provide evidence for early community-based transmission following the initial introductions of the virus. We then catalogued 13 novel mutations in the SARS-CoV-2 isolates from Moroccan patients. Interestingly, the recurrent missense variant $A>G$ at position 23,403 in the spike gene, known to be associated with virus severity, has been

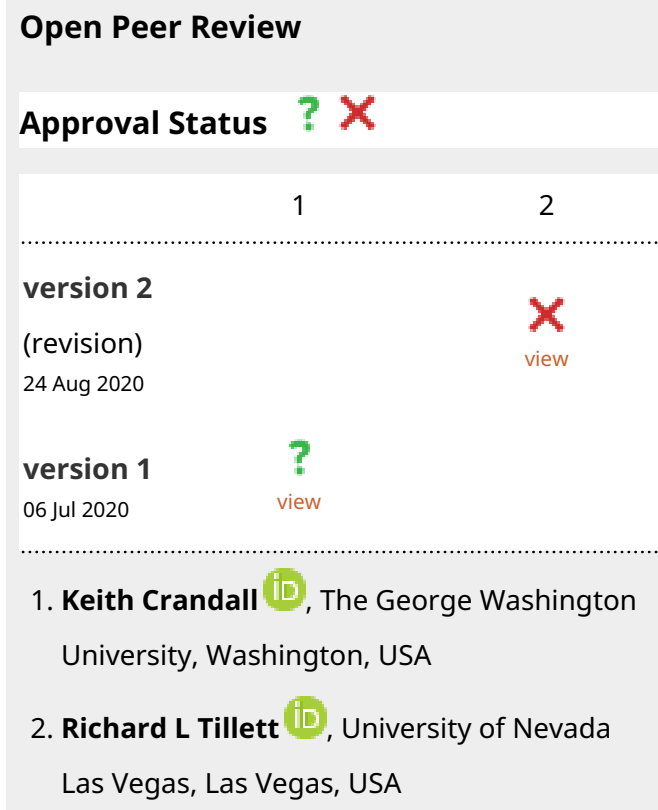

Any reports and responses or comments on the article can be found at the end of the article. 
identified in all Moroccan isolates.

Conclusions: These primary findings testify of the importance of the genomic surveillance strategies as a means of understanding the virus spread dynamics, counteracting the pandemic outbreak, and drawing useful lessons for dealing with any future emerging infectious pathogens.

\section{Keywords}

Coronavirus, SARS-CoV-2, Covid-19, Morocco, Molecular Epidemiology, Genomics Surveillance, Phylogenetics, Variant Network Analysis

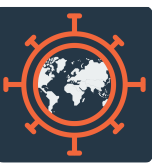

This article is included in the Emerging Diseases

and Outbreaks gateway.

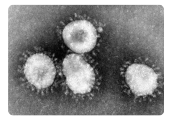

This article is included in the Coronavirus

collection.

Corresponding author: Hassan Ghazal (hassan.ghazal@fulbrightmail.org)

Author roles: Chaouni B: Data Curation, Formal Analysis, Investigation, Methodology, Visualization, Writing - Original Draft Preparation, Writing - Review \& Editing; Allali I: Conceptualization, Data Curation, Formal Analysis, Investigation, Validation, Writing Review \& Editing; Sehli S: Data Curation, Formal Analysis, Investigation, Methodology, Visualization, Writing - Original Draft Preparation; Rhalem W: Formal Analysis, Investigation, Methodology, Software, Visualization; Idrissi Azami A: Data Curation, Formal Analysis, Investigation, Methodology, Software, Validation, Visualization; Habib N: Data Curation, Formal Analysis, Investigation, Methodology, Visualization; Hamdi S: Validation, Writing - Review \& Editing; Wakrim L: Conceptualization, Writing - Review \& Editing; Bakkali A: Conceptualization, Methodology, Writing - Review \& Editing; Mahmoud M: Writing - Review \& Editing; Bouguenouch L:

Conceptualization, Methodology, Writing - Review \& Editing; Al Idrissi N: Conceptualization, Methodology, Project Administration, Writing - Review \& Editing; Bakri Y: Conceptualization, Supervision, Writing - Review \& Editing; Amzazi S: Conceptualization, Writing Review \& Editing; Barrijal S: Conceptualization, Methodology, Writing - Review \& Editing; Ghazal H: Conceptualization, Data Curation, Formal Analysis, Investigation, Methodology, Project Administration, Resources, Supervision, Validation, Visualization, Writing - Original Draft Preparation, Writing - Review \& Editing

Competing interests: No competing interests were disclosed.

Grant information: The author(s) declared that no grants were involved in supporting this work.

Copyright: $\odot 2020$ Chaouni B et al. This is an open access article distributed under the terms of the Creative Commons Attribution License, which permits unrestricted use, distribution, and reproduction in any medium, provided the original work is properly cited.

How to cite this article: Chaouni B, Allali I, Sehli S et al. Genomic evidence of multiple SARS-CoV-2 introductions into Morocco [version 1; peer review: 1 approved with reservations] F1000Research 2020, 9:679 https://doi.org/10.12688/f1000research.24810.1

First published: 06 Jul 2020, 9:679 https://doi.org/10.12688/f1000research.24810.1 


\section{Introduction}

Following its onset in December 2019, several cases of a new respiratory illness were reported in the city of Wuhan, Hubei province, China ${ }^{1}$. The disease, named coronavirus disease 2019 (COVID-19), was later on confirmed as caused by a novel coronavirus that was subsequently called the severe acute respiratory syndrome-coronavirus- $2(\mathrm{SARS}-\mathrm{CoV}-2)^{2}$. On the $12^{\text {th }}$ of March 2020, the ongoing SARS-CoV-2 outbreak was declared as a pandemic by the World Health Organization (WHO) ${ }^{3}$. As of June $16^{\text {th }} 2020$, there have been $8,228,025$ confirmed cases with 444,442 deaths around the world (John Hopkins Center $(\mathrm{JHC}))^{4}$. Currently, Morocco has reported 8931 confirmed cases and 212 deaths associated with COVID-19 (JHC update of June $16^{\text {th }}$ 2020). To gain further understanding of the molecular epidemiology of the outbreak in Morocco, we conducted a phylogenetic and a Variant Network Analyses of the full-genome sequences of 21 SARS-CoV-2 strains, 19 were isolated from Covid-19 patients in Morocco, one sequence was isolated from a patient in Melilla and another one was isolated from a Moroccan patient in Cadiz, Andalusia.

\section{Methods}

Data set

The Morocco related-sequences used in this study have been deposited in the GISAID database by the Laboratory of the Royal Gendarmerie of Morocco (LRAM) (one sequence), the Pasteur Institute of Morocco (IPM) (17 sequences), the Anoual Laboratory in Casablanca (one sequence), and the SeqCovid Spanish Project (two sequences). The sequences were collected from the GISAID database and analyzed in comparison with 500 selected genomes, that were collected between the $15^{\text {th }}$ of February 2020 (2 weeks before the first detected case in Morocco) and the $30^{\text {th }}$ of March 2020 (2 weeks after the international borders of Morocco were closed; lockdown). Only high-quality sequences have been included in this study. All sequences and metadata are provided as Extended data ${ }^{5}$ and can be found at the GitHub repository https://github.com/covidmor/ covid19_morocco.

\section{Variant calling and network analysis}

The Moroccan sequences were mapped to the Wuhan reference genome (NC_045512) using Bowtie 2.3.5.16. Variants were called using mpileup 1.7 and bcftools $1.9^{7}$. The variants were annotated using the China National Center for Bioinformation Annotator. Variant network analysis was performed using Gephi 0.9.2.

\section{Phylogenetic analysis}

A total of 500 sequences from different countries were retrieved from the GISAID database (www.gisaid.org) and aligned using Muscle 3.8.1551 multiple sequence alignment ${ }^{8}$; see Extended data, metadata ${ }^{5}$ for details on each. A maximum likelihood model was created using RaxML 8.2.12 ${ }^{9}$ with a bootstrap of 100 using the Wuhan reference genome (NC_045512) as outgroup. The phylogenetic tree was generated using Figtree $1.4 .4^{10}$.

\section{Results and discussion}

Phylogenetic tree analysis

To put the complete genomes of SARS-CoV-2 that were isolated from 19 Moroccan patients, a patient from Melilla and a Moroccan patient from Cadiz (Andalusia, Spain), into the context of the global pandemic, they were aligned together with the dataset of 500 SARS-CoV-2 complete genomes from different countries. Extended data, Figure $1^{5}$ shows the estimated maximum likelihood phylogeny. To have a good presentation of the phylogeny, we created a circular presentation subset of 120 sequences from our dataset (collected from $18^{\text {th }}$ of February 2020 (15 days before the first Moroccan case) to $15^{\text {th }}$ of March 2020 (Date of closing international borders in Morocco)) (Figure 1b).

The extended phylogenetic tree is subdivided into seven clades that correspond to the main seven SARS-CoV-2 strain types GR (red), GH (green), G (yellow), S (pink), V (magenta), L (light gray) and $\mathrm{O}$ (others; dark gray). The four strain types $\mathrm{S}, \mathrm{G}$, $\mathrm{GR}$, and GH are scattered all over the world (Extended data, Figure 2) ${ }^{5}$. According to GISAID (update of the $12^{\text {th }}$ of June 2020), they made their first appearance in February 2020, being $\mathrm{G}$ the strain type that gave origin to the GH and GR ones, while the S strain was mainly found in $\operatorname{Spain}^{11}$.

Zooming into the trees (Extended data, Figure 1) $)^{5}$, Figure 1a shows how the Moroccan isolates group into four independent clusters. In the GR clade, the sequences GISAID IDs: EPI_ISL_459973, EPI_ISL_459966 and EPI_ISL_459965 (the first infected patient in Morocco) cluster together with sequences from Italy (GISAID ID: EPI_ISL_417922), Thailand (GISAID ID: EPI_ISL_430837), Mexico (GISAID ID: EPI_ISL_452139), and Iceland (GISAID ID: EPI_ISL_ 417829) (Figure 1a: A1-3). Interestingly, the hosts of these four sequences were all travelling in Italy during the same period (Extended data, Metadata). This suggests that the first Moroccan patient (GISAID: EPI_ISP_459965), that returned to Morocco from recent travel to Bergamo, Lombardy (the most affected region in Italy), and the three other individuals from Mexico, Iceland and Thailand (GISAID: EPI_ISL_ 452139, EPI_ISL_417829 and EPI_ISL_430837, respectively) were probably infected by the same person in Italy (GISAID: EPI_ISL_417922). From this cluster, we infer, based on the sample collection dates (Extended data, Metadata) ${ }^{5}$, that the first Moroccan patient (GISAID: EPI_ISP_459965) probably infected the two other Moroccan patients whose infecting virus sequences are found in the same cluster (GISAID: EPI_ISL_459973 and EPI_ISL_459966). The Moroccan sequences (GISAID IDs: EPI_ISL_458150, EPI_ISL_459980 and EPI_ISL_459984) that cluster together in the GR clade, are close to sequences from Iceland (GISAID IDs: EPI_ISL_417861, EPI_ISL_417863 and EPI_ISL_417852) whose carriers have travel history to Italy. Furthermore, all these three Icelandic sequences are related to one Italian sequence (GISAID ID: EPI_ISL_460081). For their part, the Moroccan sequences EPI_ISL_459983, EPI_ISL_459977 and EPI_ISL_459978 cluster with another sequence from Italy (GISAID ID: EPI_ISL_452186). Surprisingly, many sequences from the GR clade were isolated from patients with known travel history to Italy (Figure 1a: A1-3). Therefore, we can conclude that all the viral sequences in this clade, where 9 Moroccan viral sequences clustered, were of Italian origin.

The four Moroccan sequences that cluster together within the G type strain (GISAID IDs: EPI_ISL_459968, EPI_ISL_459974, 
a

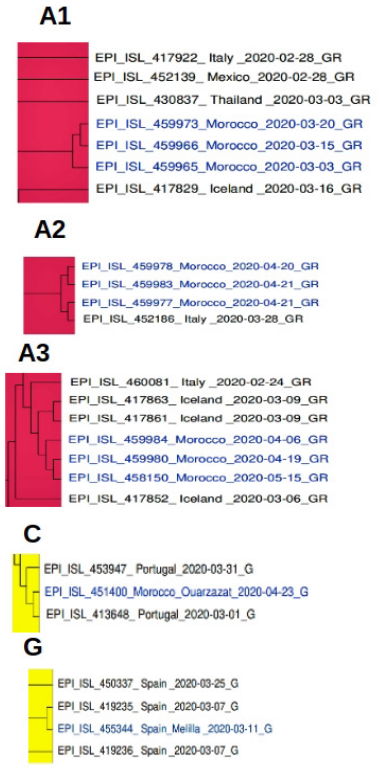

B

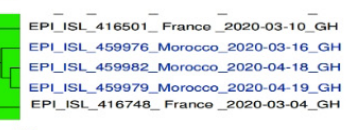
EPLISL_459979_Morocoo_2020-04-18_GH
EPI_ISL_416748_France 2020 D

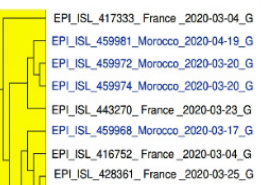
E

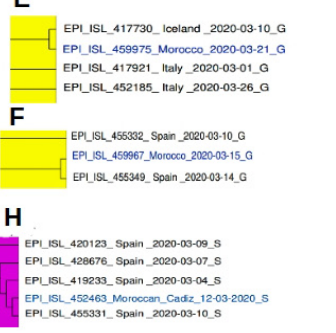

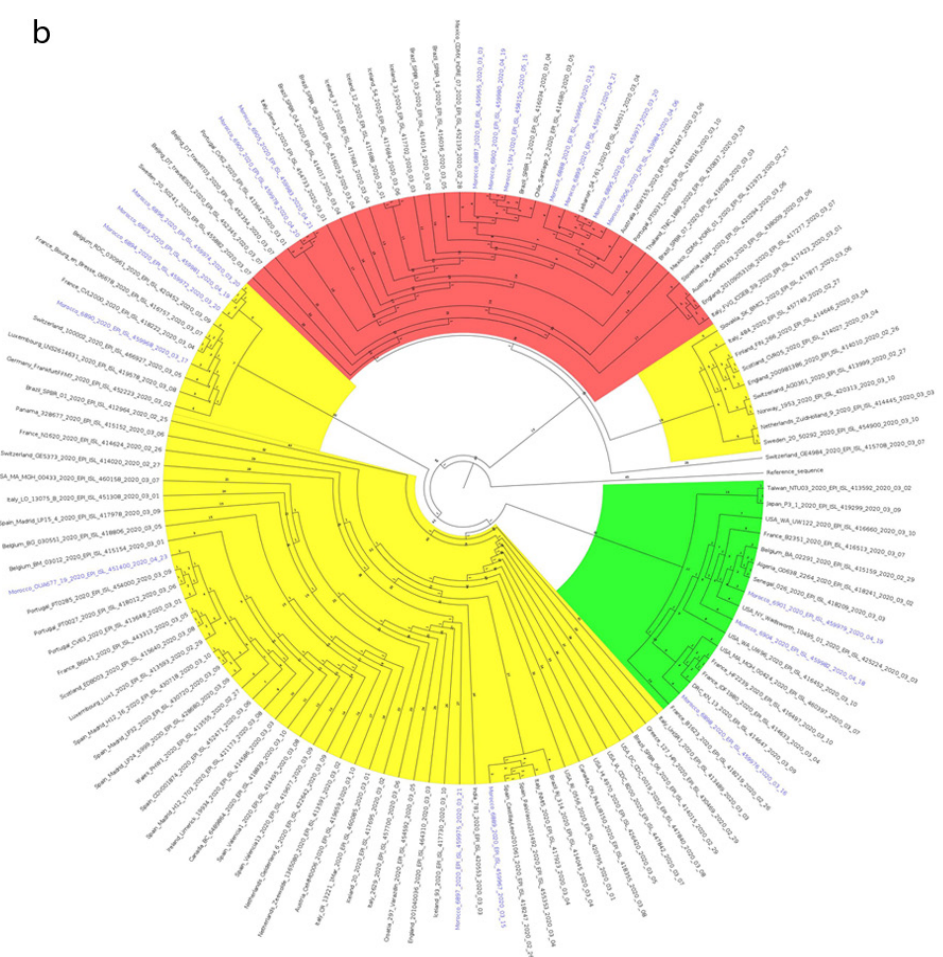

Figure 1. Phylogenetic tree analysis. (a) Maximum likelihood phylogeny, constructed using complete SARS-CoV-2 genomes from the GISAID database. The blue sequences represent the Moroccan isolates (19), a strain isolated from Melilla and a strain isolated from a Moroccan patient in Cadiz, Andalusia. The phylogenetic tree is divided into four clades: the red part for the GR strains, the green one for the GH strains, the yellow one for the $\mathrm{G}$ strains, and the pink one for the $\mathrm{S}$ strain. This Figure represents selected sub-tree extracts from the extended phylogenetic tree in Extended data, Figure $1^{5}$. A1-3: represent clusters of Moroccan strains originated from Italy and belonging to the GR clade; B: represents a cluster of Moroccan strains from a French origin and belonging to the GH clade; C: represents the Moroccan strain isolated from Ouarzazate, it originated from Portugal and belongs to the G clade; D: represents a cluster of Moroccan strains originated from France and belonging to the $\mathrm{G}$ clade; $\mathrm{E}$ : represents a Moroccan strain that originated from Italy and it belongs to the $\mathrm{G}$ clade; F: represents a Moroccan strain from a Spanish origin and belonging to the $\mathrm{G}$ clade, G: represents the sequence isolated from Melilla belonging to the $\mathrm{G}$ clade with a Spanish origin, H: represents the sequence isolated from Cadiz; it belongs to the S clade and it's close to strains from Spain. (b) Circular representation of the phylogenetic tree of a subset of 120 sequences collected before closing Moroccan international borders, constructed using complete SARS-CoV-2 genomes from the GISAID database. The blue sequences represent the Moroccan isolates (19). The phylogenetic tree is divided into three clades: the red part for the GR strains, the green one for the GH strains, and the yellow one for the $\mathrm{G}$ strains. The clade $\mathrm{S}$ containing the Cadiz sequence is absent as we removed this sequence from this phylogenetic analysis.

EPI_ISL_459972 and EPI_ISL_459981) were close to French sequences (GISAID ID: EPI_ISL_417333, EPI_ISL_443270 and EPI_ISL_416752). These four Moroccan sequences were collected the $17^{\text {th }}$ of March, the $20^{\text {th }}$ of March, the $20^{\text {th }}$ of March and the $19^{\text {th }}$ of April 2020, respectively. Interestingly, the third Covid-19 case in Morocco was a French male tourist, in his 30s that arrived in Morocco on the $7^{\text {th }}$ of March 2020 as reported by the Moroccan Health authorities. He, therefore, was most likely the source of the Moroccan viral strains that belong to the $\mathrm{G}$ type. The case reported on the $19^{\text {th }}$ of April 2020 (sequence with the GISAID ID: EPI_ISL_416752) seems to be the result of a local community transmission.

The Moroccan strain isolated from Ouarzazate (South of Morocco) (GISAID ID: EPI_ISL_451400) is close to the Portuguese sequences (GISAID ID: EPI_ISL_413648 and EPI_ISL_453947) in the G type suggesting a third introduction path to Morocco from Portugal. For their part, the Moroccan sequence (GISAID ID: EPI_ISL_459967), that also belongs to the $G$ type, is close to the Spanish sequences (GISAID IDs: EPI_ISL_455349 and EPI_ISL_455332) making Spain another point of introduction to Morocco. Furthermore, the Moroccan sequence (GISAID ID: EPI_ISL_459975) is closely related to the Italian G strain (GISAID: EPI_ISL_417921), which represents another Italian introduction to Morocco. It's also similar to a sequence from Iceland (GISAID ID: EPI_ISL_417730), where the host patient has travel history to Italy (Figure 1a: E) and could have been either infected in Italy or by another asymptomatic carrier coming from there.

Three other viral sequences isolated from Moroccan patients (GISAID IDs: EPI_ISL_459976, EPI_ISL_459982 and EPI_ISL_459979) appear in the clade of the GH strain types 


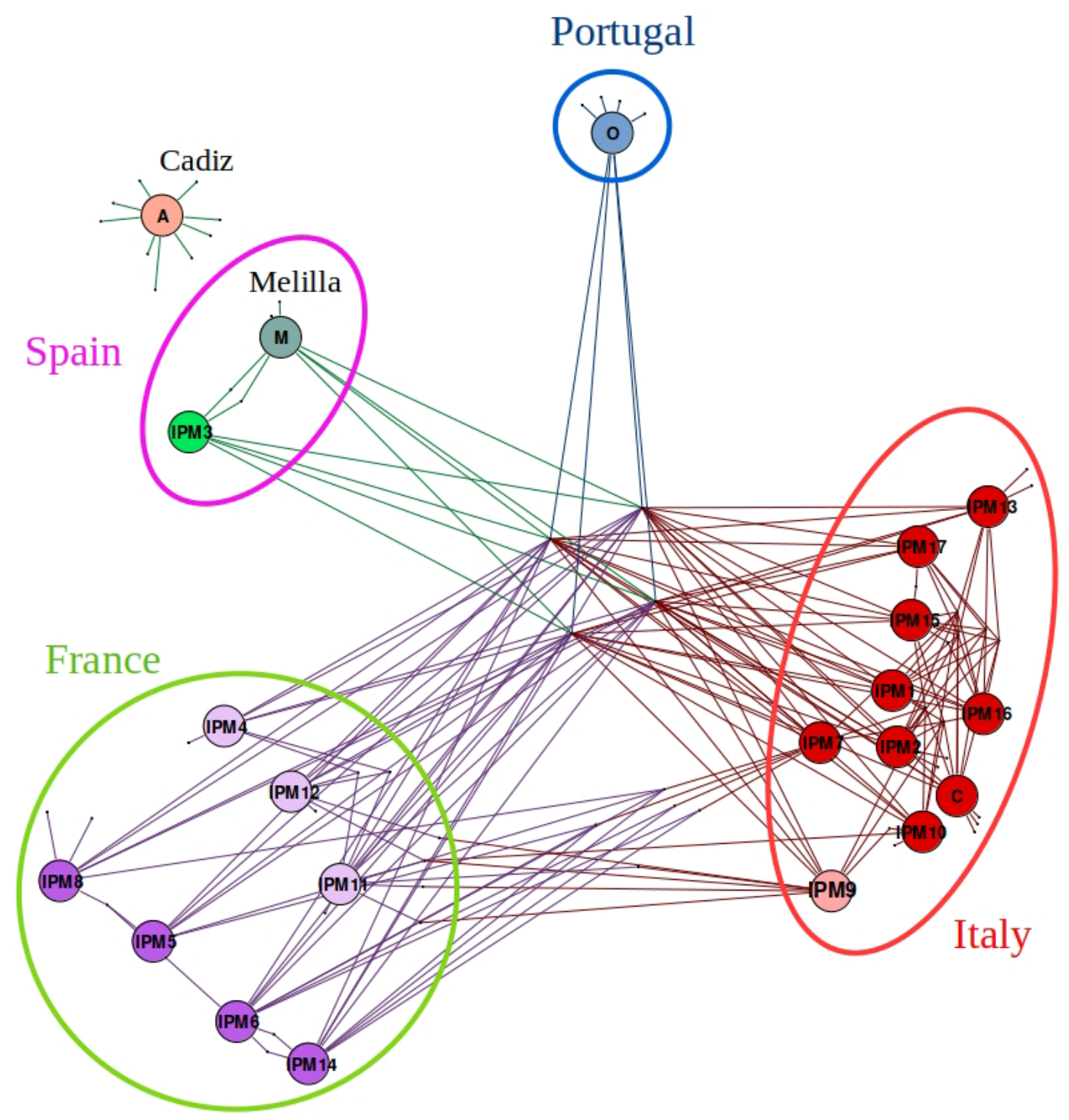

Figure 2. Variant network analysis of the 19 Moroccan genome sequences, one sequence from Melilla and another one from a Moroccan host in Cadiz, Andalusia. Every dot represents a sequence, and each edge on the network connects, using a shared variant, a sequence to one or more other sequences. The red circle group Moroccan sequences originated from Italy, the green one group sequences originated from France, the purple one assemble sequences originated from Spain, and the blue one represents the sequence originated from Portugal; we used different color gradients to separate sequences that belong to different clades in the same cluster. A: Strain isolated from Cadiz, it belongs to the S clade and it shares no variants with Moroccan strains, including the Spanish ones. M: represents the strain isolated from Melilla, it belongs to the G clade in a cluster of Spanish sequences. IPM3: is a Moroccan strain isolated by Pasteur Institut of Morocco (IPM), it belongs to the G clade and it's coming from Spain. O: represents the strain isolated by LRAM from Ouarzazate, it's the only sequence with a Portuguese origin and it belongs to the G clade. IPM9: represents a sequence collected by IPM, it originated from Italy and belongs to G clade. C; IPM1; IPM2; IPM7; IPM10; IPM13; IPM15-17: represent a strain isolated by Anoual laboratory in Casablanca (C) and sequences collected by IPM, they belong to the GR clade and they all are from Italian origin. IPM4; IPM11-12: represent strains isolated by IPM, they originated from France and they belong to the GH clade. IPM5-6; IPM8; IPM14: represent strains isolated by IPM they are from a French origin and they belong to the $\mathrm{G}$ clade.

where they cluster with French sequences (GISAID IDs: EPI_ISL_ 416748 and EPI_ISL_416501) (Figure 1a: B) suggesting a further introduction to Morocco, once more from France.

The viral sequence isolated from a patient in Melilla (GISAID ID: EPI_ISL_455344) is clustered within the G type, with Spanish sequences (GISAID IDs: EPI_ISL_419235,
EPI_ISL_450337 and EPI_ISL_419236). While the sequence isolated from a Moroccan patient in Cadiz, Andalusia (GISAID ID: EPI_ISL_452463) (Figure 1a: G) that belongs to the S type, also clusters with Spanish sequences; however, this sequence is quite far from the other Spanish related sequences. Thus, supporting the apparent neutrality of the host's genetics for the transmission and potential selection on the type of strain. 
Finally, similarity analysis of the Moroccan sequence (GISAID ID: EPI_ISL_467299) collected on May 21 $1^{\text {st }}, 2020$ and sequenced by the LRAM Laboratory, is close to the first identified French cluster, suggesting that the virus strain circulating in Morocco did not experience any major mutations and, once again, confirms the local community-based transmission of the virus. This sequence was not included in the overall analysis, as it was deposited when the writing of this work was finalizing.

Thus, the phylogenetic analysis shows that the virus outbreak in Morocco was likely the result of multiple introductions. We can highlight four independent introductions to Morocco from Italy, France, Spain, and Portugal. This finding has obvious implications for the epidemiological tracing of the pathogenic agent's initial introduction that caused the current outbreak in Morocco. Generally, the viral sequences isolated from Moroccan patients showed close relationships primarily to European strains. The geographic nearness and tourist and migratory connections, therefore, play a key role in the spread of the virus. We caution that further analyses are needed to evaluate the statistical robustness of the interference suggested herein.

\section{Network analysis}

To further understand the evolution of the SARS-CoV-2 virus within the Moroccan population and trace the infection pathways, we performed a Variant Network Analysis using Gephi. We used the complete sequences of the 21 SARS-CoV2 genomes, 19 from Morocco, and two sequences from Melilla and Andalucia (Figure 2). The variant network in Figure 2 shows three main clusters, one isolated sequence from Portugal and another one from Cadiz sharing no variants with the other sequences. The first cluster comprising the following viral strains, all closely related to each other and diagnosed in Casablanca (west Morocco) (GISAID ID: EPI_ISL_458150), IPM_1 (GISAID ID: EPI_ISL_459965), IPM_2 (GISAID ID: EPI_ISL_459966), IPM_13 (GISAID ID: EPI_ISL_459980), IPM_15 (GISAID ID: EPI_ISL_459978), IPM_7 (GISAID ID: EPI_ISL_459973), IPM_16 (GISAID ID: EPI_ISL_459977), IPM_10 (GISAID ID: EPI_ISL_459984) and IPM_17 (GISAID ID: EPI_ISL_459983) were closely related to each other. This is in agreement with the phylogenetic tree where all these sequences belong to the GR clade and appear to have been originated from Italy (Figure 1 and Extended data, Figure 1) ${ }^{5}$. The second cluster (Blue) made out of the sequences labeled IPM_5 (GISAID ID: EPI_ISL_459974), IPM_6 (GISAID ID: EPI_ISL_459968), IPM_8 (GISAID ID: EPI_ISL_459972) and IPM_14 (GISAID ID: EPI_ISL_459981) belong to the clade $\mathrm{G}$ and has a French sequence (GISAID ID: EPI_ISL_418222) as a root in the phylogenetic tree. The sequences IPM_4 (GISAID ID: EPI_ISL_459976), IPM_11 (GISAID ID: EPI_ISL_459982) and IPM_12 (GISAID ID: EPI_ISL_459979) belong to the GH clade and seem to have originated from another French sequence (GISAID: EPS_ISL_ 418219). Interestingly, this sequence belongs to the $G$ clade and is close to a strain that was isolated from an Icelandic patient that was most probably infected during his stay in Italy (GISAID ID: EPI_ISL_417730). Another separated Moroccan sequence, labelled IPM_3 (GISAID ID: EPI_ISL_459967), is closer to Spanish sequences (GISAID ID: EPI_ISL_455349, EPI_ISL_455332). The sequence of the viral strain from Ouarzazate (GISAID ID: EPI_ISL_451400) is closely related to a Portuguese sequence (GISAID ID: EPI_ISL_413648), just as shown in the phylogenetic tree. Yet, all these sequences belong to the $\mathrm{G}$ clade, which has an Italian sequence as a root. Hence, the Variant Network Analysis is in accordance with the phylogenetic tree and further supports our deduction of multiple SARS-CoV-2 introductions to Morocco from at least four European countries. Thus, while geography marks the variation that the virus undergoes in a way that we can identify geographically separated strains, population interconnection, via travel and migratory flows, seems more important than the geographical nearness for the worldwide spread of the virus.

To further test the multiple introductions to Morocco from the aforementioned four European countries, we performed a Variant Network analysis using all the Moroccan sequences as one group and compared them with randomly selected sequences from different countries (Italy, France, Spain and Portugal, in addition to Austria, Australia, and Brazil). These results show that the sequences detected during the early stages of the epidemic in Moroccan share variants with Italian (seven variants), French (four), Portuguese (three), and Spanish (three) strains. They also share one variant with strains from Austria, one with Brazil and another one with Australia (Figure 3). Interestingly, there was one shared variant between all countries located in the Spike gene $(23403 \mathrm{~A}>\mathrm{G})$ which induces the amino acid change D614G; it began spreading in Europe in early February and, when introduced to new regions, it rapidly became the dominant form. This mutation is suspected to increase the transmissibility of the virus ${ }^{12}$.

\section{Variant calling}

The genome-wide SNPs and the corresponding amino-acid positions and variations of the virus proteins are described in Table 1. Our results showed that all 20 sequences including the sequence coming from the Melilla patient, shared four mutations in common and 13 novel mutations exclusively present in the 19 sequences isolated in Morocco (Table 2). The four common mutations happened to be the same recurrent mutations described in several reports ${ }^{13}$. The mutation in the leader sequence $(241 \mathrm{C}>\mathrm{T})$ is one of the most common mutations in the SARS-CoV-2 genome; it affects an important genomic site for discontinuous sub-genomic replication. The mutation in the 5'UTR genomic region co-evolved with two other mutations, $14408 \mathrm{C}>\mathrm{T}$ and $23403 \mathrm{~A}>\mathrm{G}$. They both affect critical RNA replication proteins $(241 \mathrm{C}>\mathrm{T}, 14408 \mathrm{C}>\mathrm{T})$ and the ACE2 receptor binding protein $S(23403 \mathrm{~A}>\mathrm{G})$. We noticed that these four mutations are prevalent in the virus isolates from Europe, where the infections seem to be more severe. In fact, Yin et al. ${ }^{14}$ suggested that these mutations could increase the transmissibility of the virus. The sharing of these mutations between European and Moroccan isolates further indicates that strains circulating in Morocco came mainly from Europe. Moreover, at least one variant of the Moroccan sequences of SARS-CoV-2 is shared with one of the other countries (Table 3). The mutations found in the sequences identified in Morocco, and the identification of amino acid change, should be further 


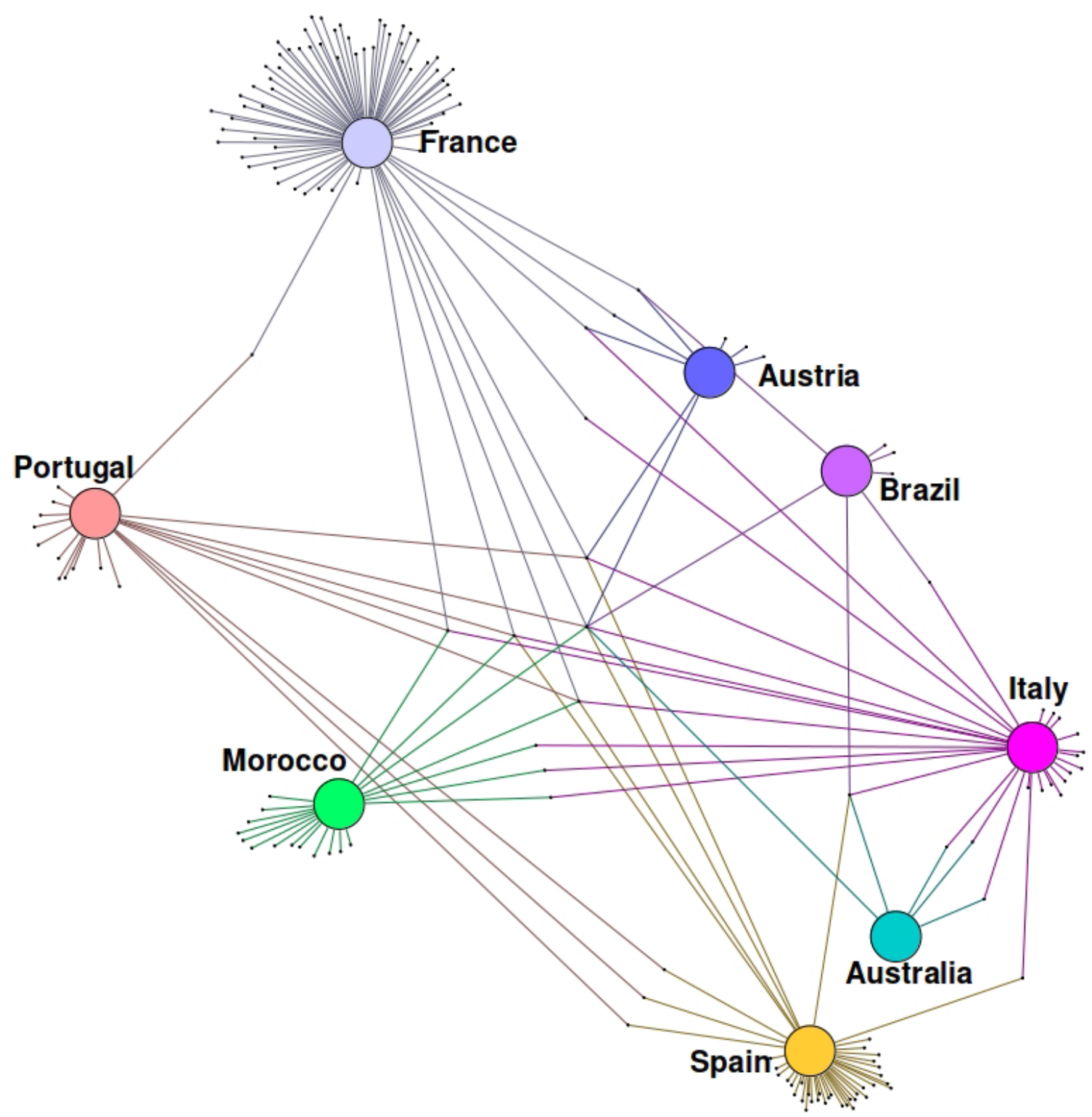

Figure 3. Variant network analysis elucidating the shared variants between sequences from different countries. Each dot represents a group of 19 sequences randomly selected from each country. The Moroccan node represents all 19 sequences isolated in Morocco. Morocco shares more variants with Italy (seven), France (four), Portugal (three) and Spain (three) than with Brazil (one), Austria (one) and Australia (one).

Table 1. List of the common mutations in all 19 sequences of SARS-CoV-2 from Morocco that are shared with European strains.

\begin{tabular}{|l|l|l|l|}
\hline Mutation & Position & Genomic region & Type of mutation \\
\hline C>T & 241 & 5'UTR & upstream_gene_variant \\
\hline C>T & 3037 & ORF1ab & Synonymous_variant \\
\hline C > T & 14408 & ORF1ab & Missense_variant \\
\hline A > G & 23403 & Gene S & Missense_variant \\
\hline
\end{tabular}

investigated in order to understand whether they affect the transmission and clinical characteristics of the SARS-CoV-2 virus circulating in Morocco.

\section{Conclusions}

Phylogenetic and variant network analyses of SARS-CoV-2 sequences from early patients with COVID-19 in Morocco showed multiple spatiotemporal introductions, primarily from Italy (ten), France (seven), Spain (one) and Portugal (one). The results also provide evidence for early community-based transmission. A variant calling analysis allowed us to catalogue new 


\title{
Table 2. List of the mutations exclusively found in the 19 \\ Moroccan sequences of SARS-CoV-2.
}

\begin{tabular}{|l|l|l|l|}
\hline Mutation & Position & Genomic region & Type of mutation \\
\hline C $>$ T & 3874 & ORF1ab & Synonymous variant \\
\hline C $>$ T & 5497 & ORF1ab & Synonymous_variant \\
\hline C $>$ T & 8175 & ORF1ab & Missense_variant \\
\hline C $>$ T & 12100 & ORF1ab & Synonymous_variant \\
\hline G $>$ T & 15380 & ORF1ab & Synonymous_variant \\
\hline C $>$ T & 18508 & ORF1ab & Missense_variant \\
\hline C $>$ T & 21513 & ORF1ab & Synonymous_variant \\
\hline C $>$ T & 26559 & Gene M & Missense_variant \\
\hline A $>$ C & 691 & ORF1ab & Synonymous_variant \\
\hline G $>$ A & 198 & 5'UTR & Upstream gene variant \\
\hline T>A & 28738 & Gene M & Synonymous_variant \\
\hline T>A & 4011 & ORF1ab & Missense_variant \\
\hline C $>$ T & 313 & ORF1ab & Synonymous_variant \\
\hline
\end{tabular}

\begin{abstract}
Table 3. List of exclusively Moroccan (M: Morocco) variants shared with at least one of the other studied countries (F: France; S: Spain; P: Portugal; I: Italy; A: Australia; Au: Austria; B: Brazil).
\end{abstract}

\begin{tabular}{|l|l|l|l|l|}
\hline Mutation & Position & Countries & Type of mutation & Genomic region \\
\hline C $>$ T & 241 & M, F, I, P, S & Upstream_gene_variant & 5'UTR \\
\hline C $>$ T & 3037 & M,F,I & Synonymous_variant & ORF1ab \\
\hline C > T & 14408 & M, F, I, P, S & Missense_variant & ORF1ab \\
\hline A > G & 23403 & M, F, I, P, S, B, A, Au & Missense_variant & Gene S \\
\hline G>C & 28883 & M, I & Missense_variant & Gene-N \\
\hline G>A & 28881 & M, I & Missense_variant & Gene-N \\
\hline G>A & 28882 & M, I & Synonymous_variant & Gene-N \\
\hline
\end{tabular}

mutations in SARS-CoV-2 isolates from Morocco. Interestingly, the recurrent missense variant $A>G$ at position 23,403 in the spike gene known to be associated with virus severity has been identified in all Moroccan isolates. However, the lack of demographic and clinical information on most of the sequences of the Moroccan isolates deposited in the database prevented us from inferring the potential link between the mutations and clinical effects of the strains. Also, the number of Moroccan genomes available at the time of this analysis hampers robust statistical inferences. Still, the results of the present work offer interesting insights into how the virus got into Morocco and primary lessons as to understanding the dynamics of the initial introductions and local transmission of SARS-CoV-2 in Morocco. This being a global pandemic, the conclusions of this study, taken together with those of recent similar studies such as those done in Egypt ${ }^{15}$, South Africa ${ }^{16}$ and Brazil ${ }^{11}$, might be applicable to other developing countries. The results of the methodology of genomic surveillance that we developed, and the methodology itself would be helpful in the case of resurgence or the emergence of any new pandemic-causing pathogen. Integrative analysis of SARS-CoV-2 should promote our understanding of the virus dynamics and interactions with hosts and environments. The method can even be further extended to reconstruct the evolutionary origins of the enhanced pathogenicity of SARS-CoV-2 and other coronaviruses that are severe human pathogens ${ }^{17}$.

\section{Data availability}

\section{Source data}

Sequences analyzed in this study were downloaded from the GISAID database. The identity of each sequence is given in the Extended data, Metadata file ${ }^{5}$. 


\section{Extended data}

Zenodo: Genomic evidence of multiple SARS-CoV-2 introductions into Morocco. http://doi.org/10.5281/zenodo. $3909463^{5}$.

This project contains the following extended data:

- Extended data Figure 1 (PDF). (Full size image of the phylogenetic tree seen in Figure 1.)

- Extended data Figure 2 (PNG). (Full genome tree derived from all outbreak sequences.)
- Extended data Labels (PDF). (Labels of the Moroccan sequences)

- Extended data Metadata (PDF). (Metadata for the sequences assessed in this study.)

All supplementary material and figures can be found at GitHub: https://github.com/covidmor/covid19_morocco.

Extended data are available under the terms of the Creative Commons Zero "No rights reserved" data waiver (CCO 1.0 Public domain dedication).
1. Wu P, Hao X, Lau EHY, et al:: Real-time tentative assessment of the epidemiological characteristics of novel coronavirus infections in Wuhan, China, as at 22 January 2020. Euro Surveill. 2020; 25(3): 2000044 PubMed Abstract | Publisher Full Text | Free Full Text

2. Uddin M, Mustafa F, Rizvi TA, et al:: SARS-CoV-2/CovID-19: Viral Genomics, Epidemiology, Vaccines, and Therapeutic Interventions. Viruses. 2020; 12(5) 526. [cited 2020 Jun 15].

PubMed Abstract | Publisher Full Text | Free Full Text

3. WHO announces COVID-19 outbreak a pandemic. 2020. [cited 2020 Jun 15]. Reference Source

4. Johns Hopkins Center: Coronavirus COVID-19 (2019-nCoV). 2020. [cited 2020 Jun 16].

Reference Source

5. covidmor: covidmor/covid19_morocco: Genomic evidence of multiple SARSCoV-2 introductions into Morocco (Version 1.0). Zenodo. 2020. http://www.doi.org/10.5281/zenodo.3909463

6. Langmead B, Salzberg SL: Fast gapped-read alignment with Bowtie 2. Nat Methods. 2012; 9(4): 357-9.

PubMed Abstract | Publisher Full Text | Free Full Text

7. Li H, Handsaker B, Wysoker A, et al:: The Sequence Alignment/Map format and SAMtools. Bioinformatics. 2009; 25(16): 2078-9.

PubMed Abstract | Publisher Full Text | Free Full Text

8. Edgar RC: MUSCLE: multiple sequence alignment with high accuracy and high throughput. Nucleic Acids Res. 2004; 32(5): 1792-7. PubMed Abstract | Publisher Full Text | Free Full Text

9. Stamatakis A: RAxML-VI-HPC: maximum likelihood-based phylogenetic analyses with thousands of taxa and mixed models. Bioinformatics. 2006; 22(21): 2688-90.

PubMed Abstract | Publisher Full Text
10. Rambaut A: FigTree Version 1.4.4. 2020. [cited 2020 Jun 15] Reference Source

11. de Jesus JG, Sacchi C, Claro I, et al.: First cases of coronavirus disease (COVID-19) in Brazil, South America. 2020. Reference Source

12. Korber B, Fischer WM, Gnanakaran S, et al.: Spike mutation pipeline reveals the emergence of a more transmissible form of SARS-CoV-2. bioRxiv. 2020; 2020.04.29.069054. Publisher Full Text

13. Pachetti M, Marini B, Benedetti F, et al.: Emerging SARS-CoV-2 mutation hot spots include a novel RNA-dependent-RNA polymerase variant. $J$ Transl Med. 2020; 18(1): 179. [cited 2020 Jun 4].

PubMed Abstract | Publisher Full Text | Free Full Text

14. Yin C: Genotyping coronavirus SARS-CoV-2: methods and implications. Genomics. 2020. [cited 2020 Jun 15]. PubMed Abstract | Publisher Full Text | Free Full Tex

15. Kandeil A, Mostafa A, El-Shesheny R, et al:: Coding-Complete Genome Sequences of Two SARS-CoV-2 Isolates from Egypt. Microbiol Resour Announc. 2020; 9(22): e00489-20. [cited 2020 Jun 15]. PubMed Abstract | Publisher Full Text | Free Full Tex

16. Mavian C, Pond SK, Marini S, et al.: Sampling bias and incorrect rooting make phylogenetic network tracing of SARS-COV-2 infections unreliable. Proc Natl Acad Sci U S A. 2020; 117(23): 12522-12523. PubMed Abstract | Publisher Full Text | Free Full Text

17. Gussow AB, Auslander N, Faure G, et al:: Genomic determinants of pathogenicity in SARS-CoV-2 and other human coronaviruses. bioRxiv. 2020; 2020.04.05.026450.

PubMed Abstract | Publisher Full Text | Free Full Text 


\section{Open Peer Review}

\section{Current Peer Review Status: ?}

\section{Version 1}

Reviewer Report 13 July 2020

https://doi.org/10.5256/f1000research.27373.r66513

(c) 2020 Crandall K. This is an open access peer review report distributed under the terms of the Creative Commons Attribution License, which permits unrestricted use, distribution, and reproduction in any medium, provided the original work is properly cited.

\section{Keith Crandall}

Computational Biology Institute, Milken Institute School of Public Health, The George Washington University, Washington, DC, 20052, USA

Summary: This paper reports on new sequence data on the SARS-CoV-2 outbreak in Morocco. Through phylogenetic analyses they identify 4 distinct introductions to Morocco from distinct European origins followed by local transmissions. The analysis are OK, but need some additional work (see below). Specifically, there are no bootstrap analyses discussed to provide confidence in the resulting phylogenetic relationships (the main result of the paper). The authors use an excellent sampling strategy, focusing on Moroccan sequences and then those available from two weeks before the first known case in Morocco and two weeks after the closing of the boarders. Their data are deposited with GISAID and thereby publicly available (with some effort and approval), but they also provide their entire working set on GitHub. Excellent! This is the way to do open science which is so critical for the COVID studies. However, the nomenclatural approach is confusing and the phylogenetic presentation could use some help. There are a lot of different things going on with this analysis (lots of different metadata to focus on) and the authors could do a better job of making things more clear in their presentation. Below are some ideas to help with both the analyses and interpretation.

Abstract/Results spike gene variant associated 'with virus severity' - this doesn't make sense to me. What does it mean to have a severe virus? Do you mean severity in clinical outcomes?

Methods/Data Set - you say 500 sequences were selected from GISAID both before and after the initial case in Morocco. I cannot, however, get a feel for the relative split or the relative timeline. It would be really helpful to have a graph that had time along the $x$-axis (perhaps by week or something) and then different colors for the pre-GISAID, post-GISAID, and Moroccan sequences.

Methods/Phylogenetic analysis - the authors state the 'model was created using RaxML'. First, RAxML doesn't create models. It estimates phylogenies. Second, it needs a model to do this. What model was used in this analysis? There is an updated version of ModelTest, ModelTest-NG https://academic.oup.com/mbe/article/37/1/291/5552155 that helps with optimizing a model of 
evolution for a data set to then feed into RAXML. I recommend the authors use this tool to justify a model choice, report the model, and then use it for the ML estimate. Then 'FigTree' doesn't 'generate' a tree, it is a tool for phylogeny visualization. I would recommend $\mathrm{TTOL}$ as an alternative. iTOL also allows for more legible mapping of metadata on the phylogeny: https://itol.embl.de/ Given that the phylogeny seems to be the main result, the authors should conduct a bootstrap analysis with at least 1000 replicates and show bootstrap support values on the phylogeny as an assessment of confidence.

In general, no details of the analyses are provided and it is therefore impossible to replicate the study. The authors simply list software. They need to be more explicit about how they actually ran their analyses.

Some pretty specific information is provided on patients, e.g., 'French male tourist' with a date of the case report. I'm wondering if these individuals are consented to participate in the research and be effectively identified. This seems pretty specific. There is no information about consent.

Results/Phylogenetic analyses - the phylogenetic results are difficult to read. The image is low resolution, so if I zoom in on the pdf, it just gets blurry. There are a different nomenclatures being thrown about (A1, A1, GR, GH, etc.) and it is hard to find these groups on the tree and or their significance. There are no bootstrap values, so there is no confidence in the presented relationships. Again, if the authors us iTOL, they can make a more effective presentation with better labels on the tree. I don't see the pink clade on the main tree at all.

In the text, everything is presented in terms of GISAID accessions, which is nice for track but not for presentation. It would be better if the authors relabeled things in the supplementary table with more accessible labels like Moroc1, Franc1, Spain1, etc., and then not list every isolate in a clade in the text. It would be even more informative if you included isolation date in the name, e.g., Moroc1_3_21. This would allow readers to immediately interpret the phylogeny. As it is, it is uninformative with respect to isolation date and difficult to identify isolates from different countries.

Then in the sequence variant network analysis, the nomenclature seems changed again. Now there are things called 'IPMx'. I don't understand why this network analysis is not done in the context of the other non-Moroccan sequences. Given the phylogenetic result that the Moroccan sequences don't form a cluster by themselves, but rather cluster with other sequences with distinct geographic origins, it makes no sense to analyze them by themselves here. You are just picking up variants that differentiate the geographic clades and assigning them (incorrectly) to the Moroccan sequences. It would be much more powerful to compare the Moroccan sequences to the others WITHIN EACH CLADE as this allows for effectively independent replicates of viral adaptation to Moroccan hosts. Then you can look for convergently evolved changes associated with the Moroccan isolates.

Tables 1, 2, \& 3 should identify the nonsynonymous changes (e.g., show the amino acid replacement in the same way you show the nucleotide substitution).

\section{Is the work clearly and accurately presented and does it cite the current literature?} Partly 
Is the study design appropriate and is the work technically sound?

Partly

Are sufficient details of methods and analysis provided to allow replication by others? Partly

If applicable, is the statistical analysis and its interpretation appropriate? Partly

Are all the source data underlying the results available to ensure full reproducibility? Yes

Are the conclusions drawn adequately supported by the results?

Partly

Competing Interests: No competing interests were disclosed.

Reviewer Expertise: Phylogenetics, population genetics, molecular evolution, bioinformatics, infectious disease

I confirm that I have read this submission and believe that I have an appropriate level of expertise to confirm that it is of an acceptable scientific standard, however I have significant reservations, as outlined above.

The benefits of publishing with F1000Research:

- Your article is published within days, with no editorial bias

- You can publish traditional articles, null/negative results, case reports, data notes and more

- The peer review process is transparent and collaborative

- Your article is indexed in PubMed after passing peer review

- Dedicated customer support at every stage

For pre-submission enquiries, contact research@f1000.com 